\title{
Research on Synchronous Grid-Connected of Micro-grid by Using Kalman Filter
}

\author{
Xiaobing Xu ${ }^{1, *}$, Bing $\mathrm{Li}^{1}$, Jianping Wang ${ }^{1}$, Chengqiang Zheng ${ }^{1}$, Xiaojia Qin ${ }^{1}$, Wei Sun ${ }^{1}$ and Qin Wang ${ }^{2}$ \\ ${ }^{1}$ HeFei University of Technology, China \\ ${ }^{2}$ JiangSu TongLin Electric Co., Ltd, China \\ *Corresponding author
}

\begin{abstract}
The coordinating control of distributed power and smoothly grid-connected strategy is the key to well grid-connected. In this paper, a strategy of synchronous grid-connected control based on Kalman filter estimation is put forward. By calculating the difference between the previous and the present state, the next state can be estimated whether it meets the requirements of grid-connected, and the micro-grid connected to grid is fulfilled quickly and smoothly. The simulation shows the validity of the proposed scheme.
\end{abstract} Keywords-kalman filter; micro-grid; synchronous
grid-connected

\section{INTRODUCTION}

Research and application of micro-grid have become popular focus in recent years [1-2]. When a micro-grid is synchronously connected to the large power grid, the key research point is to choose a smooth grid connected control strategy by measuring data on both sides of the grid and coordinated control of the distributed power supply.

Some scholars have studied the control method of micro-grid and synchronous grid-connected strategies. It is described [3] that the micro-grid can be safely connected based on master-slave control whereas the algorithm is too complex. A droop control method without link line is used to realize the access of micro-grid smoothly, but it lack of the accuracy of grid-connected time according to the paper [4]. The multi-loop control strategy of distributed power inverter used in micro-grid is researched in paper [5], but it did not take the load change into account. The control method of low voltage micro-grid based on droop control characteristic is proposed to avoid the change process of control strategy but extend the time of grid-connected as referred to [6].

A smooth grid connected control method of the micro-grid with multiple distributed power is discussed in this paper. First a synchronous controller is built in a typical micro-grid model, and the three-phase voltage signals of micro-grid and main grid are pretreated. Second, calculate the difference of amplitude, phase and frequency. Then synchronous grid-connected time can be estimated by using kalman filter method for the difference. Finally, simulation is given, and the error comparison to the difference estimation of voltage signals is carried out. The results show the effectiveness of estimating grid-connected based on kalman filter.

\section{The Model And CONTRol Methods of MCrio-Grid}

Figure I shows the typical model of micro-grid, including some distributed power like diesel generating sets,photovoltaic, wind power and some local load [8]. The improved peer-to-peer control is the coordination control method for the system. The diesel adopts isochronous control to keep stability for the frequency and voltage of micro-grid. The photovoltaic array and the wind power generator employ PQ control, with maximum power point tracing module. The synchronous controller is used to control that micro-grid connect to main grid. The switch will be closed and grid-connected will be realized when meeting grid-connected condition.

Figure II shows the model of synchronous controller. The detected three-phase voltage signals of main grid and micro-grid after the filtering and signal conditioner, and then the amplitude, frequency, phase will be calculated by PLL. The difference between both sides is compared to judge whether meet the grid-connected condition. If meet, the breaker will get a trigger signal and micro-grid is connected to grid, or the diesel generator will acquire a compensating signal of the difference value. It quickly meet the grid-connected condition by adjusting the frequency and voltage rely on the diesel generator.

\section{Synchronous Signals Detecting AND PRETREATMENT}

Because of the extensive use of power electronic devices, the randomness and intermittent of renewable energy are hooked up to grid, and a large number of nonlinear loads switching in, the components of the signal in power system is becoming more and more complex. There are some phenomenon in grid like higher harmonic, three-phase voltage asymmetry and so on. The more seriousness is that infecting the precision of signal measurement. So it is necessary to filter the signal, adjust the three-phase voltage's sequence and other pretreatment. 


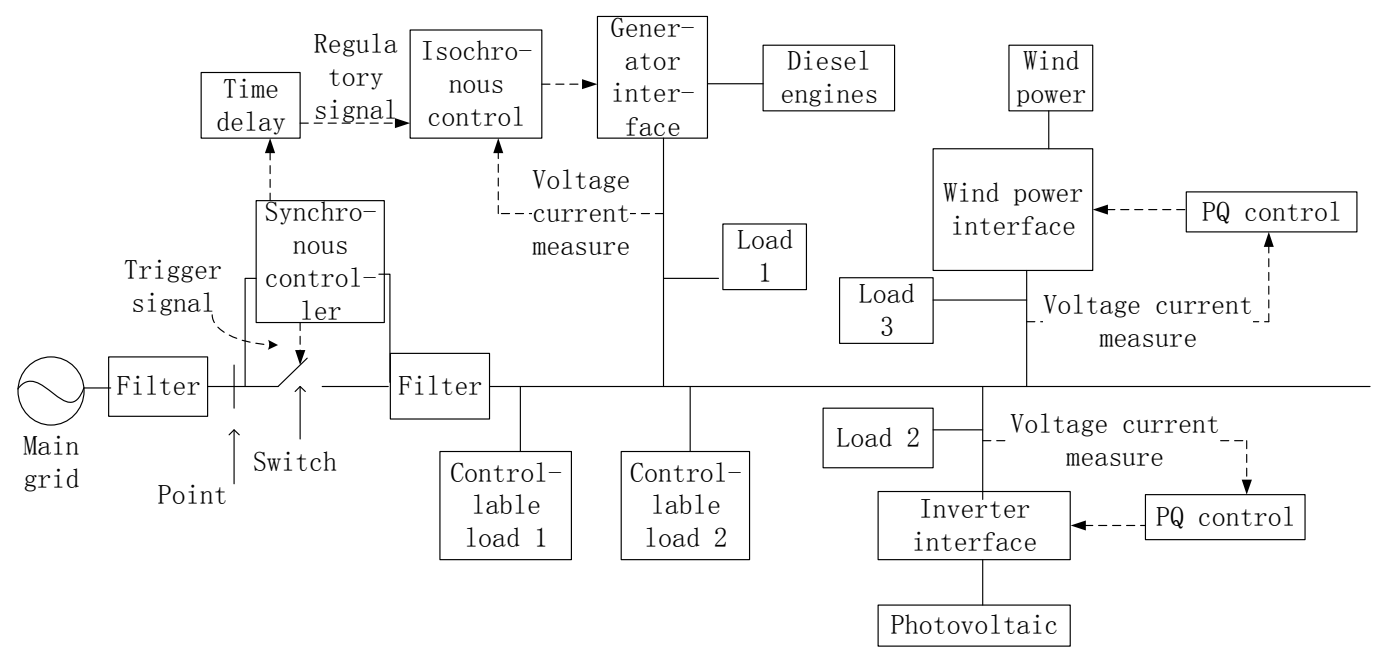

FIGURE I. TYPICAL MODEL OF MICRO-GRID

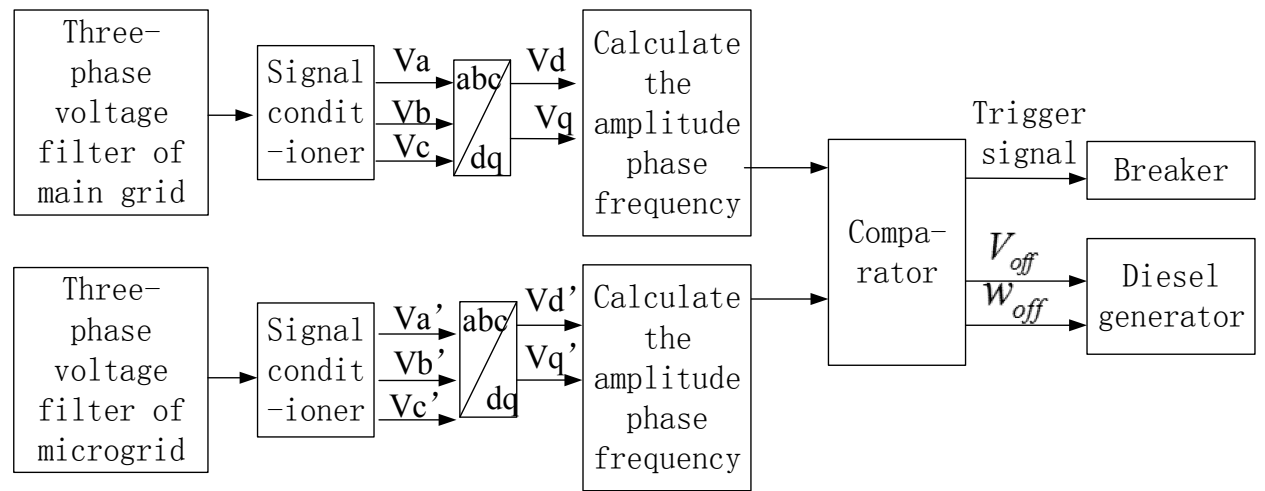

FIGURE II. SYNCHRONOUS CONTROLLER MODEL STRUCTURE

\section{A. Voltage Filter and Parameter Selection}

In this paper, we have added a LC voltage filter before synchronous controller [10]:

The inductance can be designed as follows:

$$
\frac{\left(2 U_{\alpha k}-3 E_{m}\right) E_{m} T_{s}}{2 U_{\alpha k} \Delta i_{\max }} \leq L \leq \frac{2 U_{\alpha k}}{3 I_{m} w}
$$

Where $U_{a k}$ is DC bus voltage (V), $E_{m}$ is AC voltage amplitude (V), $T_{s}$ is switch period (s), $\Delta i_{\max }$ is Maximum ripple current of filter inductance.

The capacitance can be designed by fundamental frequency and carrier frequency of system as follows:

$$
\left\{\begin{array}{l}
10 f_{n} \leq f_{0} \leq \frac{f_{s}}{10} \\
f_{0}=1 /(2 \pi \sqrt{L C})
\end{array}\right.
$$

Where $f_{n}$ is fundamental frequency, $f_{s}$ is carrier frequency, $f_{0}$ is resonance frequency.

\section{B. Virtual Three-phase Voltage Conditioner}

Because of the loads asymmetry and the distributed generation, the three-phase voltage will be asymmetric. So we added a signal conditioner to solve it. We can build a virtual three-phase symmetric voltage signal that the amplitude is same as the original signal by positive-sequence phasor. The amplitude and phase can be precisely calculated by means of the virtual three-phase symmetric voltage signal [9].

The three-phase asymmetry, and positive-sequence voltage can be calculated as follows:

$$
V_{1}=\frac{1}{3}\left(V_{a}+a V_{b}+a^{2} V_{c}\right)
$$

Where $a=1 \angle 120^{\circ}=e^{j(2 / 3) \pi}$

According to the positive-sequence voltage and the phase operator,we can create symmetry virtual three-phase voltage instead of the original three-phase voltage as follows:

$$
\left[\begin{array}{ccc}
\tilde{v_{a}} & \tilde{v_{b}} & \tilde{v_{c}}
\end{array}\right]=\left[\begin{array}{lll}
1 & a^{2} & a
\end{array}\right]^{T} \cdot V_{1}
$$




\section{The Method of Pre-Synchronization CONTROL BASED ON KALMAN FILTER}

After pretreatment of synchronous signal, in order to meet the grid-connected condition, we need to calculate the amplitude difference, phase difference and frequency difference of the main grid and micro-grid. Because of the breaker closing time delay between the main grid and micro-grid, we should consider the breaker closing time delay when estimate the difference value. Due to the randomness of the distributed generation, it is difficult to predict the difference, but kalman filter can make it.

Kalman filter is supply to the linear system that exist random factors, we take the phase difference as an example to build kalman filter:

1) Two-dimensional state vector :

$x_{k}=\left[\begin{array}{cc}\Delta \theta_{k} & \dot{\Delta} \theta_{k}\end{array}\right]^{T}$ where $\Delta \theta_{k}$ phase difference of $(\mathrm{k}) \mathrm{th}$, $\Delta \dot{\theta}_{k}$ the first derivative,slip angle frequency.

\section{2) State equation of the system}

The changing process of phase-angle of the system can be described by the following equation:

$$
x_{k+1}=A x_{k}+\Gamma \lambda_{k}
$$

Where $\mathrm{A}, \Gamma$ is constant matrix, $\lambda_{k}$ is system noise, a zero mean Gauss white noise sequence.

3) Measurement equation of the system:

$$
v_{k}=C_{k}+\mu_{k}
$$

Where $\mathrm{C}$ is $1, v_{k}$ is one-dimensional measurement vector, $\mu_{k}$ is measurement error,a zero mean Gauss noise.

\section{4) System initial state:}

The initial phase difference: $\hat{x}_{0 \mid 0}=E\left(x_{0}\right)$

Estimation error covariance matrix: $P_{0,0}=\operatorname{Var}\left(x_{0}\right)$
Noise covariance matrix of measurement equation: $R_{k}=\mathrm{E}$ $\left\{\mu_{k}^{\wedge} 2\right\}$

Covariance matrix of system noise: $Q_{k}=\mathrm{E}\left\{\lambda_{k}^{\wedge} 2\right\}$

5) Step prediction derivation:

According to prediction model of kalman filter theory, step prediction derivation as follows:

$$
\begin{gathered}
P_{1,0}=A P_{0,0} A^{T}+\Gamma Q_{k} \Gamma^{T} \\
G_{1}=P_{1,0} C^{T}\left(C P_{1,0} C^{T}+R_{k}\right)^{-1} \\
P_{1,1}=\left(I-G_{1} C\right) P_{1,0}
\end{gathered}
$$

So we can get the predicted value of the next state:

$$
\hat{x}_{1 \mid 1}=A \hat{x}_{0 \mid 0}+G_{1}\left(v_{k}-C A \hat{x}_{0 \mid 0}\right)
$$

By the equation(5) (10), the rest can be deduced: $x_{2 \mid 2}$, $X_{3 \mid 3}, \ldots, X_{k \mid k}, X_{k+1 \mid k+1}, \ldots$

Where $G_{k}$ is kalman gain $(\mathrm{k})$ th, a state estimation(k)th.

We can find from the iterative process that the state estimation of phase difference is connected with the present input and the last state estimation of phase difference. So only save the last state estimation of phase difference can be realized real-time processing. For the random dynamic system, the next state value can be estimated quickly based on the last state value. It meet the grid-connected condition.

\section{The Simulation of The Grid CONNECTING SYNCHRONOUSLY}

We can build the simulation model of micro-grid on Matlab Simulink platform. And comparing the result of simulation between the estimation arithmetic based on kalman filter and difference method. There are including diesel generator, photovoltaic power system, wind power system, breaker, synchronous controller, main grid and loads in the simulation model.

Figure III shows the interior structure of synchronous controller. 


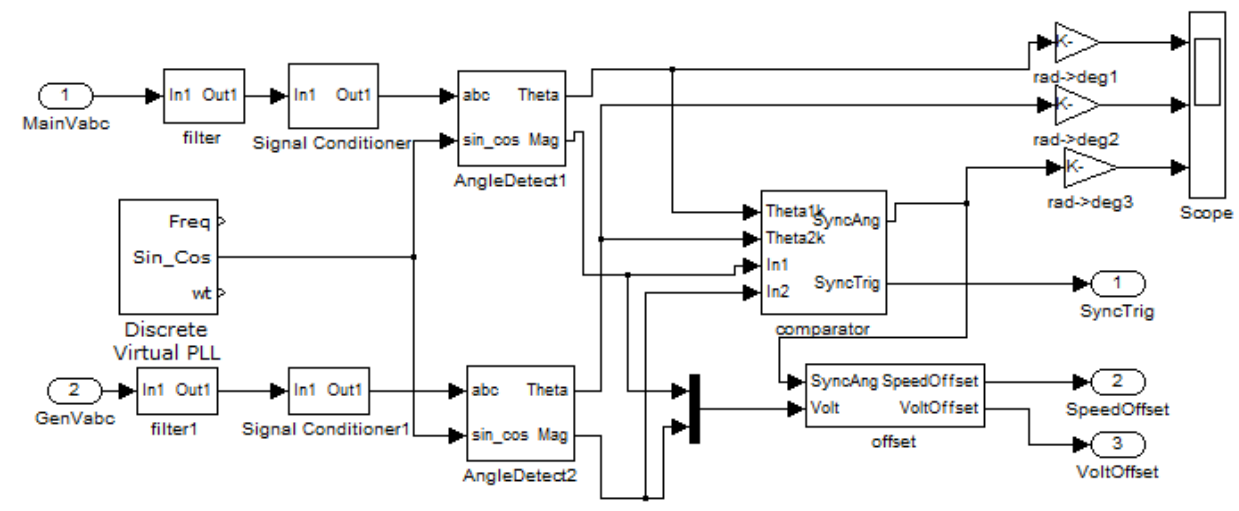

FIGURE III. SIMULATION MODEL OF SYNCHRONOUS CONTROLLER

The initial parameter setting of simulation experiments based on kalman filter estimation algorithm and difference method estimation as the following:

Grid:bus voltage is $380 \mathrm{~V}$; photovoltaic power system work at maximum power point, rated capacity is $10 \mathrm{kVA}$; wind power keep constant power operation; rated capacity of diesel generator is $50 \mathrm{kVA}$; load1:active power is $20 \mathrm{kVA}$; load2: active power is $100 \mathrm{~W}$, reactive power is $1 \mathrm{kVar}$; controllable load1: power is $5 \mathrm{~kW}$; controllable load2: power is $3 \mathrm{kVar}$.

The micro-grid system consist of diesel generator, photovoltaic power, wind power, load is linked to main grid with $22.9 \mathrm{kV}, 50 \mathrm{~Hz}$ by breaker and transform. The phase difference can be estimated by difference method estimation and kalman filter estimation algorithm. The simulation analysis of micro-grid connect to grid as follows:

At first, the micro-grid is running at island state, and the equipment states are:the diesel generator join in, the photovoltaic join in, the wind power join in, load1 join in, load 2 join in, controllable load1 join in, controllable load 2 join in; cut off the controllable load1 at piont $\mathrm{A}$; cut off the controllable load 2 at point $\mathrm{B}$; at point $\mathrm{C}$, the micro-grid meet the grid-connected condition and turn to grid-connected operation; the breaker closing time relay is $30 \mathrm{~ms}$.

Figure IV shows the frequency of micro-grid based on kalman filter estimatied method. Figure V shows the change of bus voltage. Where Fg is main grid frequency. The frequency and voltage of micro-grid will be changed with the change of load. At the stage of $0 \sim \mathrm{C}$, the frequency and voltage of micro-grid adjust and estimate continuously, until meet the requirement of grid-connected at point $\mathrm{C}$.

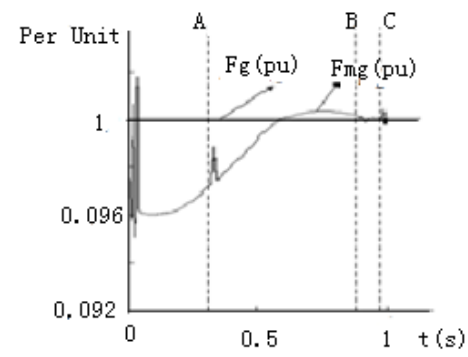

FIGURE IV. FREQUENCY OF MICRO-GRID AND GRID

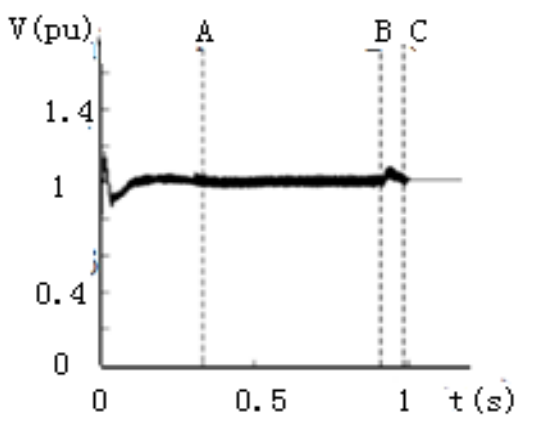

FIGURE V. BUS VOLTAGE OF MICRO-GRID

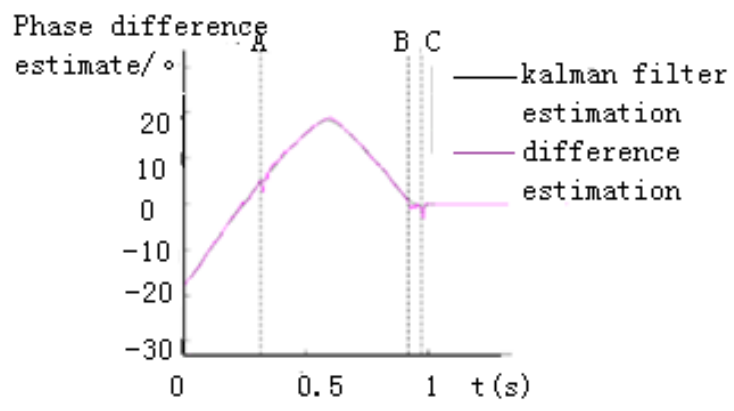

FIGURE VI. PHASE DIFFERENCE ESTIMATION

Figure VI shows that both the difference method estimation and the kalman filter estimated algorithm can estimate the phase difference, but for the change load the difference method estimation cannot well estimate, because it have an obvious oscillation that cause delay for grid-connected. The kalman filter can estimate the change of phase difference precisely, and realize the micro-grid connect to main grid smoothly.

\section{CONCLUSION}

This paper put forward a smooth micro-grid control strategy based on kalman filter estimation algorithm with some distributed power in micro-grid system, and that realize grid-connected quickly and smoothly.

The simulation results shows that the method based on kalman filter is more feasible and valid than on phase 
difference. It help to improve the precisely and quickly of prediction.

\section{REFERENCE}

[1] Y W, YANG P, WANG Y W, ZHAO ZH L. Typical Characteristics and Key Technologies of Micro-grid. Automation of Electronic Power Systems, 2015,39(8):168-175.

[2] SHEN CH,WU X Y,WANG ZH W,ZHAO M,HUANG X Q.Practice and rethinking of micro-grid[J]. Power System Protection and Control, 2014,42(5):1-11.

[3] XU SH H, LI J L. Grid-connected/Island Operation Control Strategy for Photovoltaic/Battery Micro-grid[J]. Proceedings of the CSEE, 2013,33(34):25-33.

[4] ZHANG L, XU Y Q, WANG Z P, LI X D, LI P. Control Scheme of Micro-grid Fed by Synchronous Generator and Voltage Source Inverter[J]. Power System Technology, 2011,35(3):170-176.

[5] WANG CH SH, XIAO ZH X, WANG SH X. Multiple Feedback Loop Control Scheme for Inverters of the Micro Source in Micro-grids[J]. Transactions of China Electrotechnical Society, 2009,24(2):100-107

[6] JI M W, ZHANG X, YANG SH Y. Study on the control strategies of voltage source inverter in micro-grid[J]. JOURNAL OF HEFEI UNIVERSITY OF TECHNOLOGY, 2009,32(11):1678-1682.

[7] CT Lee, RP Jiang, PT Cheng, A grid synchronization method for droop-controlled distributed energy resource converters, IEEE Transaction on Industry Applications, 2011,49(2):954-962.

[8] P. Arboleya, D. Diaz, J.M. Guerrero, P. Garcia, F. Briz, C. Gonzalez-Moran, J. Gomez Aleixandre. An improved control schemc based in droop characteristic for micro-grid converter. Electric Power Syst ems Research, 2009,80(10):1215-1221.

[9] C Cho, SK Kim, JH Jeon, S Kim, New ideas for a soft synchronizer applied to CHP cogeneration, IEEE Transactins on Power Delivery, 2011,26(1):11-21.

[10] [10]QIN ZH, ZHANG H Y, XU Q, GUO Z L. Research on Filter of Voltage Inverter in Micro-grid[J]. Journal of Electric Power, 2015,30(3):243-246. 